\title{
A Model - Based Jamming Detection Method for Electro- Hydrostatic Actuators
}

Emre Murat Poyraz ${ }^{\circledR}$ Hakan Caliskan ${ }^{\circledR}$ Ali Emre Turgut ${ }^{(\infty}$

Middle East Technical University, Department of Mechanical Engineering, Ankara,Turkey

\section{A B S T R AC T}

A $\mathrm{n}$ anomaly or a fault, which may be treated as insignificant in a complicated engineering system, like an electro-hydraulic flight control actuator, can cause considerable performance degradation and deteriorating effects or even worse it may end up with a catastrophic system failure. One of the problems commonly observed in air vehicles is called jamming. Jamming is the type of failure where an actuator connected to a flight control surface permanently gets stuck at a certain position and does not move anymore. This might cause a loss of control of the air vehicle causing severe consequences. Although, there are several methods to detect jamming in the literature, still there is not a universally accepted solution. In this paper, a novel threshold-based fault detection and diagnosis method is proposed. The method is based on the variance of time rate of change of disturbance load and the average tracking error. When these two quantities are above a certain corresponding threshold, jamming is detected. The method is tested on an experimental setup. The experiments show that the detection performance of the method is satisfactory and the detection is performed under one second for all the test cases.

\section{Keywords:}

Fault detection and diagnosis; Disturbance observer; EHA; Jamming; Flight control actuation.

\section{INTRODUCTION}

$\mathrm{D}$ ue to increasing demand towards higher system reliability and safety, a great amount of research about fault detection and diagnosis are being carried out. For safety critical systems, like an aircraft, consequences of faults in a flight control system can be extremely hazardous for human life. Flight control systems mostly rely on electro hydraulic actuation technology for providing necessary power to control surfaces of aircrafts. Design and integration of a flight control systems often includes fault detection algorithms in order to make the system more robust against failures. There are many studies on fault detection and diagnosis of different faulty cases in flight control systems such as; oscillatory failure case [1], stall load [2], jamming [3], runaway [4], and incipient sensor failures [5]. One of the most important of them all is jamming. Jamming is a system failure where an actuator connected to a control surface is permanently stuck at a random position. This has several consequences such as undesirable aircraft motion, increase in drag force and fuel consumption.

Several model-based approaches for jamming detection exist in the literature. Among them, Kalman filtering and observer-based approaches are the most well- known and preferred ones. Ossmann et al. [2] proposed a model-based fault detection and diagnosis method for the actuator jamming at small surface deflections. For the detection of jamming, a discrete version of the linear parameter varying (LPV) modelling approach is proposed in [6] and; it also has the fault identification functionality as in [2]. The error residual $r(t)$ is generated based on the position output of LPV model of the hydraulic actuator. Then, the residual evaluation signal $\theta_{r}(t)$ is obtained by using a Narendra type fault evaluator [7]. The detection performances have been assessed by simulating the jamming failure scenario, during level flight and special maneuver cases. The results show a high degree of robustness in fault detection and diagnosis (FDD) for the whole range of tests and a satisfactory detection performance. Kalman filter has also been extensively used in fault detection and diagnosis applications. Goupil et al. [3] proposed a Kalman filter-based approach for jamming detection. Proposed method has received certification on the new generation Airbus A350 aircraft.

In this paper, a threshold-based FDD method is designed for an electro-hydrostatic actuator (EHA). The method is based on two indicators; variance of the time 
rate of change of disturbance load and average tracking error. One threshold for each indicator is defined and when two indicators exceed their corresponding threshold, jamming is detected by the method. In the design of the FDD method, first the linear mathematical model of the EHA is obtained. Then, the state and disturbance observers are designed. The information from the mathematical model (including the state observer) and the disturbance observer are then used in the threshold-based model. The model is also verified extensively in an experimental setup.

The main contributions of this study are: firstly, to the best of our knowledge, none of the studies about jamming focuses on the actuator dynamic. In this paper, the dynamics of the actuator is considered to extract information related to the failure. Secondly, unlike the other studies, in this paper, only the available actuator states are used to identify the jamming not the aircraft states or feedbacks as in [6]. This approach might make the integration of the developed algorithm a lot easier as the only states used for detection of jamming failures are already available as actuator feedback that is continuously processed for the position control loop. Whereas integration of aircraft state feedbacks like calibrated air speed, center of gravity position, aircraft mass and the altitude used in [6] would significantly complicate the overall integration process, requiring various sensor (air data sensor, inertial measurement unit, etc.) outputs, and need a lot more complex interface management for both hardware and software.

The paper is organized as follows: In Section 2, the method, including the mathematical modelling of the EHA, observer design and the fault identification steps, for the detection of jamming are introduced. Then in Section 3, verification of the proposed method is performed. Finally, in Section 4, the results are discussed and the conclusion is made.

\section{METHODOLOGY}

The overall methodology for the detection and identification of jamming starts with the mathematical modelling of the actuator. Later, a state and a disturbance observer are designed based on the mathematical model of the actuator. At the last step, the output of the disturbance observer is analyzed for fault detection purposes.

\section{Mathematical Modelling}

The EHA system consists of a hydraulic piston, a pump, an $\mathrm{AC}$ electric servo motor driving the pump, a shuttle valve and a hydraulic accumulator for differential flow compensation [8]. Details of physical relations within the EHA system are illustrated in [9].

There are basically two working regions for the EHA. Depending on the load pressure value, the shuttle valve adjusts its opening and lets the flow go into the accumulator and/or the actuator chambers. When the load pressure is low, about 7-8 bar, the shuttle valve opens partially to both chambers or only one of the chambers. The pilot-operated spool of the shuttle valve positions itself naturally. For the other case where the load pressure exceeds these 7-8 bar of the differential pressure, then the shuttle valve fully opens. In this configuration, one of the two chambers is connected to the accumulator and thus the accumulator and the connected chamber can be assumed to have the same pressure. Within the scope of this paper, the EHA is operated in the fully-opened shuttle valve configuration by adjusting the counter loading.

In order to develop a simplified mathematical model of the system in Fig. 1, several assumptions are made. The accumulator pressure and temperature responses are assumed to be considerably slow so that the accumulator dynamics are neglected. The electric motor current dynamics is assumed to be very fast and it is also neglected. Shuttle valve is assumed to be fully open. Therefore, spool dynamics of the shuttle valve is not considered. In a fully-opened shuttle valve condition, only one hydraulic chamber determines the pressure dynamics since the hydraulic accumulator capacitance together with the hydraulic conductance of the shuttle valve are assumed to be considerably high. In other words,

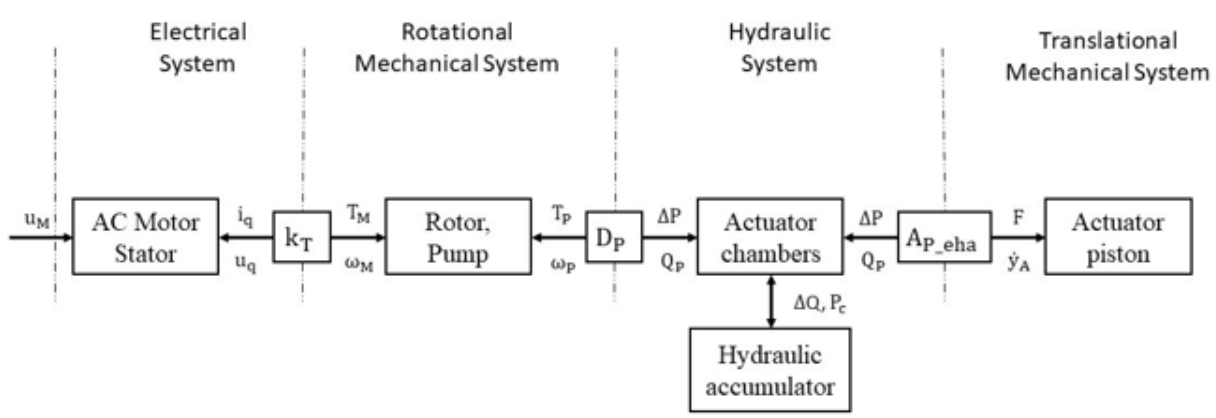

Figure 1. Components and their relations within the EHA system. 
change in the load pressure is equal to one of the chamber pressures as $\delta P_{L}=\delta P_{a}$ or $\delta P_{L}=\delta P_{b}$ depending on the open side of the shuttle valve [9].

In Fig. 1, $T_{M}$ is the torque generated by the servo motor $P_{C}$ is the accumulator pressure, $Q_{P}$ is the hydraulic flowrate between the pump and the actuator . $\Delta P$ represents the pressure difference and the $\Delta Q$ shows the hydraulic flow.

\section{Electrical and Rotational Mechanical System}

The electric motor and the hydraulic pump are assumed to be coupled through a rigid coupling. Therefore, the pump inertia together with the frictional losses is lumped into electric motor dynamics. The resulting equation of motion of the motor shaft is:

$$
k_{T} i_{q}=\left(J^{\prime}{ }_{M}\right) \dot{\omega}_{M}+\left(b^{\prime}{ }_{M}\right) \omega_{M}+D_{p}\left(P_{a}-P_{b}\right) \cdot 10^{-3}
$$

where, $J^{\prime}{ }_{M}=J_{M}+J_{P}$ and $b^{\prime}{ }_{M}=b_{M}+b_{P}$ are the total effective inertia and friction coefficients, respectively. Note that for consistency of units, the right-hand side of the equation, $D_{p}\left(\mathrm{P}_{\mathrm{a}}-\mathrm{P}_{\mathrm{b}}\right)$ term, is multiplied by $10^{-3} \cdot k_{T}$ is the electric motor torque constant. $\omega_{M}$ is the angular speed of the pump in, $J_{M}$ is the inertia of the rotor of the electric motor, $J_{P}$ is the inertia of the hydraulic pump rotor, $D_{p}$ is the pump displacement, $P_{a}$ and $P_{b}$ are piston and rod side chamber pressures, respectively, $b_{p}$ is the viscous friction coefficient of the pump and $b_{M}$ is the viscous friction coefficient of the motor.

\section{Hydraulic and Translational Mechanical System}

The equation of motion for the hydraulic actuator is:

$$
A_{p}\left(P_{a}-a P_{b}\right)=m \ddot{y}_{A}+F_{D}+F_{f}
$$

where, $y_{A}$ is the actuator piston position, $A_{P}$ is the piston cross sectional area, $m$ is the mass of the piston and the rod of the actuator, $\mathrm{a}$ is the pressure area ratio of the hydraulic cylinder, $F_{D}$ is the disturbance force acting on the actuator and $b$ is the viscous friction coefficient of the actuator.

The friction force here is modelled as:

$$
F_{f}=b \dot{y}_{A}
$$

where $\dot{y}_{A}$ is the velocity of the piston. The continuity equation for the piston and the rod side chambers can be written considering the leakage flow to be proportional to the load pressure $\left(P_{L}\right)$ as follows:

$$
\begin{aligned}
& C_{a} \dot{P}_{L}=D_{p} \omega_{M}-A_{p} \dot{y}_{A}-H P_{L} \\
& C_{b} \dot{P}_{L}=-D_{p} \omega_{M}+a A_{p} \dot{y}_{A}-H P_{L}
\end{aligned}
$$

where $H$ is the leakage flow coefficient of the pump, $C$ and $C_{b}$ are the piston side hydraulic chamber capacitance, the rod side hydraulic chamber capacitance, respectively. $\dot{P}_{L}$ is the time rate of change of load pressure.

\section{State Space Representation of the Overall System}

The state variables are defined as:

$$
\begin{aligned}
& x_{1}=y_{A} \\
& x_{2}=\dot{y}_{A} \\
& x_{3}=P_{L}=\left(\mathrm{P}_{\mathrm{a}}-\mathrm{aP}_{\mathrm{b}}\right), \\
& x_{4}=\omega_{M}
\end{aligned}
$$

In matrix form the system is:

$$
[\dot{x}]=A[x]+B[u]
$$

The input vector $\mathrm{u}$ includes $u_{M}$ the motor torque and $F_{d}$ disturbance load as:

\begin{tabular}{|c|c|c|c|}
\hline Parameter & Description & Value & Unit \\
\hline$k_{T}$ & torque constant & 1.52 & $N m / A$ \\
\hline$J M$ & $\begin{array}{l}\text { rotor inertia of the } \\
\text { electric motor }\end{array}$ & $27 \cdot 3 \cdot 10^{-4}$ & $\mathrm{~kg} \cdot \mathrm{m}^{2}$ \\
\hline$J P$ & $\begin{array}{l}\text { hydraulic pump rotor } \\
\text { inertia }\end{array}$ & $1.93 \cdot 10^{-4}$ & $\mathrm{~kg} \cdot \mathrm{m}^{2}$ \\
\hline$D p$ & pump displacement & 8 & $\mathrm{~cm}^{3} / \mathrm{rad}$ \\
\hline$b M$ & $\begin{array}{l}\text { motor viscous friction } \\
\text { coefficient }\end{array}$ & $7 \cdot 10^{-3}$ & $\mathrm{Nms} / \mathrm{rad}$ \\
\hline$b_{p_{\text {eha }}}$ & $\begin{array}{c}\text { pump viscous friction } \\
\text { coefficient }\end{array}$ & 0.035 & $\mathrm{Nms} / \mathrm{rad}$ \\
\hline
\end{tabular}

$$
\begin{aligned}
& {[u]=\left[\begin{array}{c}
u_{M} \\
F_{d}
\end{array}\right]} \\
& A=\left[\begin{array}{cccc}
0 & 1 & 0 & 0 \\
0 & -\frac{b_{p}}{m} & \frac{A_{p}}{m} & 0 \\
0 & -\frac{A_{p}}{C} & -\frac{H}{C} & \frac{D_{p}}{C} \\
0 & 0 & -\frac{D_{p}}{J^{\prime}{ }_{M}} & -\frac{b^{\prime}{ }_{M}}{J^{\prime}}
\end{array}\right] \\
& B=\left[\begin{array}{cccc}
0 & -\frac{1}{m} & 0 & 0 \\
0 & 0 & 0 & \frac{1}{j^{\prime}{ }_{M}}
\end{array}\right]^{T}
\end{aligned}
$$

The parameters used for the mathematical modelling of the electrical and rotational mechanical subsystems are shown in Table 1.

Table 1. Parameters of the electrical and rotational mechanical subsystems [9]. 
The parameters used for the mathematical modelling of the hydraulic and translational mechanical subsystems are shown in Table 2.

Table 2. Parameters of the hydraulic and translational mechanical subsystems [9].

\begin{tabular}{|c|c|c|c|}
\hline Parameter & Description & Value & Unit \\
\hline$A_{p_{-} \text {hha }}$ & $\begin{array}{c}\text { piston side cross sectional } \\
\text { area }\end{array}$ & 2827.4 & $m m^{2}$ \\
\hline$a_{\text {eha }}$ & area ratio & 0.75 & - \\
\hline$m_{\text {eha }}$ & $\begin{array}{c}\text { mass of the piston and } \\
\text { the rod }\end{array}$ & 9.6 & $\mathrm{~kg}$ \\
\hline$b_{e h a}$ & $\begin{array}{l}\text { viscous friction coefficient } \\
\text { of the actuator }\end{array}$ & 6.3 & $\mathrm{Ns} / \mathrm{mm}$ \\
\hline$C_{a}$ & $\begin{array}{l}\text { piston side hydraulic } \\
\text { chamber capacitance }\end{array}$ & 302.5 & $\mathrm{~mm}^{3} / \mathrm{s} \cdot \mathrm{MPa}$ \\
\hline$C_{b}$ & $\begin{array}{l}\text { rod side hydraulic } \\
\text { chamber capacitance }\end{array}$ & 302.5 & $\mathrm{~mm}^{3} / \mathrm{s} \cdot \mathrm{MPa}$ \\
\hline
\end{tabular}

Capacitance values of $C_{a}$ and $C_{b}$ are assumed to be constant to a value of $C$ that is calculated at the position where the two chamber volumes are equal.

\section{State and Disturbance Observer Design}

\section{State Observer}

A Luenberger observer is designed for the EHA. Using the state-space form in (12) and (13) together with the system parameters, the following matrix equations are obtained:

$$
\begin{aligned}
{[\dot{x}] } & =\left[\begin{array}{cccc}
0 & 1 & 0 & 0 \\
0 & -673.07 & 302072.465 & 0 \\
0 & -9.35 & 0 & 0.4408 \\
0 & 0 & -5369.86 & -2.39
\end{array}\right][x] \\
& +\left[\begin{array}{ccc}
0 & 0 \\
-0.107 & 0 \\
0 & 0 \\
0 & 342.465
\end{array}\right]\left[F_{d} u\right] \\
y= & {\left[\begin{array}{cccc}
1 & 0 & 0 & 0 \\
0 & 0 & 0 & 1
\end{array}\right][X]+\left[\begin{array}{ll}
0 & 0
\end{array}\right]\left[\begin{array}{c}
F_{d} \\
u
\end{array}\right] }
\end{aligned}
$$

Open loop poles of the EHA system is calculated as:

$$
\begin{aligned}
p_{0}= & 0+0 i,-336.26+1646.9 i,-336.26-1646.9 i \\
& ,-2.9594
\end{aligned}
$$

The desired poles for the observer are chosen as:

$$
\begin{aligned}
p_{c}= & -5+0 i,-400+1646.9 i,-400-1646.9 i,-3.0 \\
& +0 i
\end{aligned}
$$

Finally, the gain matrix of the observer is found using pole placement technique:

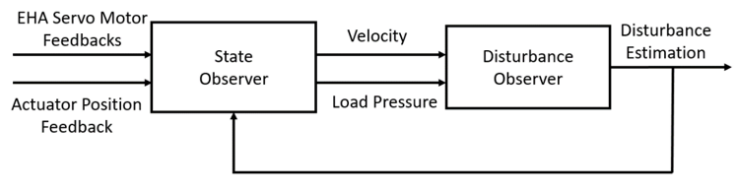

Figure 2. Observer Structure of State and Disturbance Estimations.

$$
L=\left[\begin{array}{cc}
30 & 0.5 \\
2.25 \cdot 10^{5} & 125 \\
1.2 \cdot 10^{3} & 0.1 \\
3.2 \cdot 10^{2} & 20
\end{array}\right]
$$

Eventual aim of the designed state observer is to supply unknown state information to the disturbance observer. However, the disturbance load acting on the actuator is an unknown too. Therefore, a structure including two observers working simultaneously is developed for both the state and disturbance estimations. Two observers work together with one estimating the state variables while the other estimating the disturbance as shown in Fig. 2.

\section{Disturbance Observer}

The force equilibrium on the piston can be re-written as:

$$
F_{d}=-m_{p} \ddot{x}_{p}+p_{L} A_{p}-b_{p} \dot{x}_{p}
$$

which may also be expressed in terms of state variables as:

$$
F_{d}=-m_{p} \dot{x}_{2}+A_{p} x_{3}-b_{p} x_{2}
$$

Estimation of the disturbance load is defined as $\hat{d}$ and the dynamics of this estimation with an observer gain $L_{0}$ is designed as follows: [10]

$$
\dot{\hat{d}}=-L_{1}\left(\mathrm{~m}_{\mathrm{p}} \dot{\mathrm{x}}_{2}-\mathrm{A}_{\mathrm{p}} \mathrm{x}_{3}+\mathrm{b}_{\mathrm{p}} \mathrm{x}_{2}+\hat{\mathrm{d}}\right)
$$

In Eq. 23, the derivative of the state $x_{2}$ which amplifies the noise in $x_{2}$ decreasing the estimation performance. In order to avoid this problem, an auxiliary variable $\zeta$, as represented in [11], is defined as:

$$
\zeta=-\hat{d}-L_{1} m_{p} x_{2}
$$

and the dynamics of the auxiliary variable is

$$
\dot{\zeta}=-L 1\left(\zeta+L_{1} m_{p} x_{2}\right)+\mathrm{L}_{1}\left(\mathrm{~b}_{\mathrm{p}} \mathrm{x}_{2}-\mathrm{A}_{\mathrm{p}} \mathrm{x}_{3}\right)
$$

The load pressure and the velocity estimations together with the piston position are used in the disturbance observer model. At this point, it's worth noting that the direct usage of the chamber pressure information would significantly increase the fidelity of the disturbance observer model. It is not used in this study because the pressure feedback may not always be available for a flyby-wire actuator in an aircraft [12]. 


\section{Fault Identification}

As the first step, time rate of change of the estimated disturbance load is calculated as the residual signal. A detection signal $i(t)$, in Eq. 24, is generated that triggers the fault identification process, when the variance, $\sigma$, of the disturbance rate $\dot{F}_{d_{-} \text {obs }}$ exceeds a predefined threshold. The threshold value $\mathrm{\tau}$ for jamming is chosen based on the not-jammed test cases. Jamming results in an increase in the time rate of change of the disturbance load and it also changes its variance. Using these facts, fault information could be extracted from output test data.

$$
i(t)=\left\{\begin{array}{l}
1 \text { if } \sigma\left(\dot{F}_{d_{-} o b s}\right) \geq \tau \\
0 \text { if } \sigma\left(\dot{F}_{d_{-} o b s}\right)<\tau
\end{array}\right.
$$

One possible drawback of variance computation is that it requires the storage of $\mathrm{n}$ many samples, which may not be desirable for real time operations. An alternative way is to use recursive methods for mean and variance calculation [13].

Another parameter is needed to enhance the reliability of the proposed FDD method. Since the actuator rod stays approximately constant at the jammed position, moving average $\bar{x}$ of the position tracking error may be analyzed to create a jamming indicator. A fault confirmation signal $\mu(t)$ is generated that decides the presence or absence of a fault if the moving average $\bar{x}$ of the position tracking error $|e|$ exceeds a predefined threshold. The threshold value $\tau_{j}$ for jamming cases is based on not-jammed test cases. Two threshold-based steps consolidate the fault detection function and increase the reliability of the developed FDD system.

$$
\mu(t)=\left\{\begin{array}{l}
1 \text { if } \bar{x}|e| \geq \tau_{j} \\
0 \text { if } \bar{x}|e|<\tau_{j}
\end{array}\right.
$$

where $|e|=y_{\text {reference }}-y_{\text {measured }}$ is the tracking error.

\section{EXPERIMENTAL ANALYSIS}

\section{Test Setup}

A schematic drawing of the hydraulic test bench, which was designed and constructed by Çalışkan [9] and Akova [14] is shown in Fig. 3. It consists of two hydraulic actuation systems. The one on the left is an electro hydrostatic actuation system controlled by a hydraulic pump whereas the one on the right is a conventional hydraulic system that acts as the load simulator and it is controlled by a servo proportional valve. Two systems are connected to each other via a flexible coupling mechanism with a force sensor. Closed-loop force control of the load simulator is accomplished using the force sensor. The piston position is also measured which is used in the disturbance feedforward controller as the feedback signal.

The EHA is a closed-loop position system where position tracking is achieved through a closed-loop feedback and feedforward control. An AC servo motor is placed to drive the hydraulic pump in the system. The hydraulic actuator used in the study is a single rod type hydraulic piston which creates unequal flowrates for the retraction and extension sides. Çalışkan developed a novel method to compensate this unequal flowrate difference using a hydraulic accumulator and a 3-position 3-way shuttle valve [8]. Together with the position of the piston of the EHA, speed of the motor is

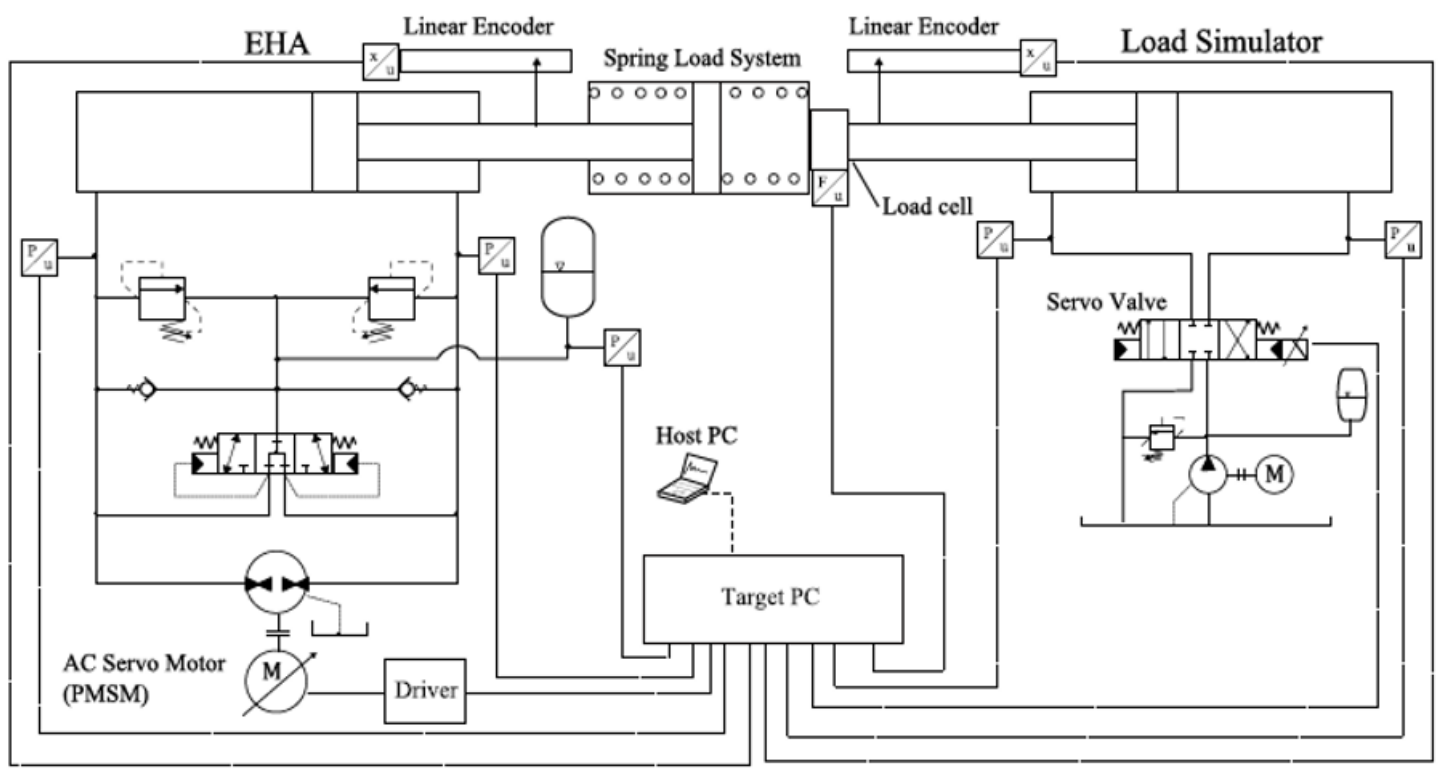

Figure 3. Schematic drawing of the hydraulic test bench [9]. 
controlled in closed-loop manner. Speed and torque of the servo motor, pressures in the two piston chambers and in the accumulator is measured simultaneously together with the actuator position.

The SpeedGoat real time target PC is equipped by IO105 analog input and IO111 analog output modules. The servo motor speed and torque, pressures of the two chambers of the EHA and accumulator, and the actuator position are measured simultaneously, with a $1 \mathrm{kHz}$ sampling frequency. The pressure transducers are made by Trafag and rated up to 250 bar with $0-10 \mathrm{~V}$ output. They are mounted on the hydraulic manifold. The EHA position is measured by a linear encoder made by ATEK. The encoder is connected to the rod of the EHA, it has $20 \mu \mathrm{m}$ grid spacing and enables $5 \mu \mathrm{m}$ resolution at $4 \mathrm{X}$ decoding. [9]. The accuracy of the pressure transducer (Trafag, 8472) is $\pm 0.5 \%$ and the load Cell (Burster Model 8524) is $\pm 0.25 \%$.

\section{Verification of the Observer Models}

A set of test data is used where the reference position of the EHA and the magnitude of the force of the load simulator are controlled as shown in Fig.s 4 and 5.

In the closed-loop tests, position of the EHA is kept constant at $100 \mathrm{~mm}$ after $\mathrm{t}=6 \mathrm{secs}$ (Fig. 4). A force signal with varying amplitude is generated as the reference input for the load simulator. Both positive and negative disturbance loads of $12 \mathrm{kN}$ are applied and its amplitude is changed between $1-2 \mathrm{kN}$ while the frequency of the square waves is varied bet-

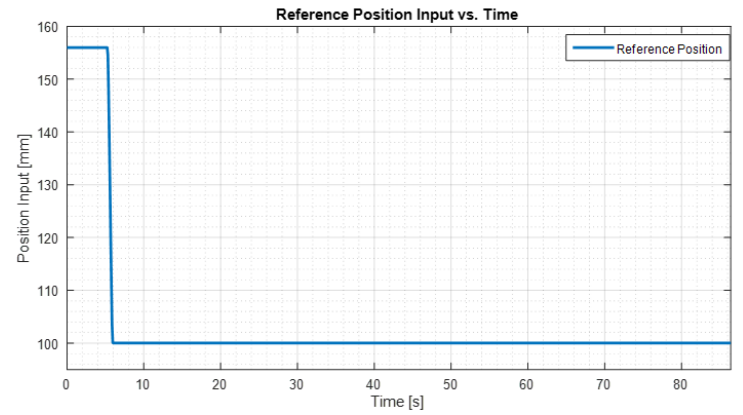

Figure 4. Reference Position Input to the EHA.

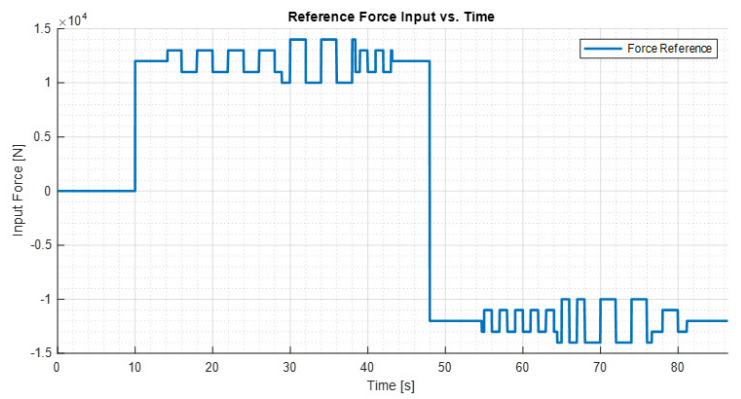

Figure 5. Reference Force Input to the Load Simulator. ween $0.5-2 \mathrm{~Hz}$ (Fig. 5). Velocity estimation via a dedicated Kalman Filter [9] is also used together with the velocity estimation output of the state observer. Some operating regions in the following test results are zoomed in to show the estimation performance of the observers. Especially, the region where the disturbance load is varied is tried to be focused on. Comparison of the estimated states and disturbances with measured feedbacks, via the transducers in the setup, is given in the following figures.

The state observer gives accurate results, being less than $0.1 \%$, for the estimation of position (Fig. 6). This is in fact an expected result since feedback is available for this state variable.

Velocity estimation (Fig. 7) shows some differences between the estimation by state observer and the estimation by a Kalman Filter [9]. This can be explained by the fact that the Kalman Filter, uses a kinematic filtering method whereas in the velocity estimation by the state observer relies more on the system dynamics. There is also a very small error in the estimation of observer for the zero-velocity region which might be overcome by increasing the related gain term in the observer gain matrix.

For the estimation of the load pressure, some undesirable peak points are observed like the ones in 32th and 34th seconds of the simulation as in Fig. 8. The main reason

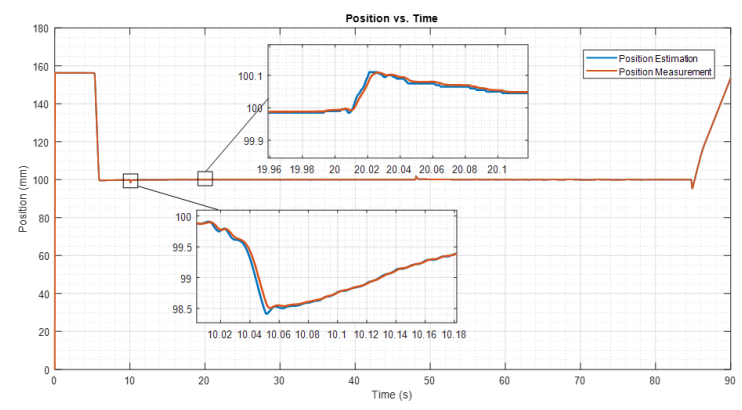

Figure 6. Measured Position Response and the Position Estimation of the EHA.

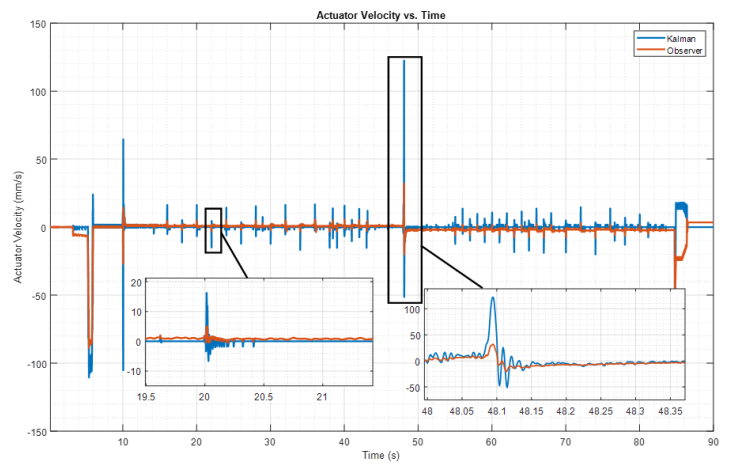

Figure 7. Kalman Filtered Velocity Estimation [9] vs. Observer based Velocity Estimation of the EHA. 


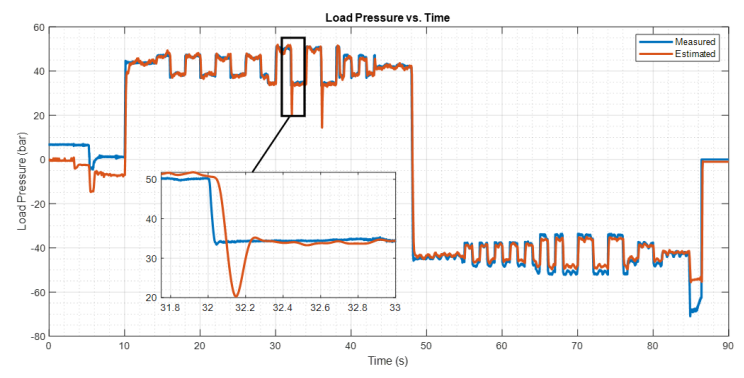

Figure 8. Measured Load Pressure and the Load Pressure Estimation of the EHA.

why such peak points are observed is due to the fact that the EHA system automatically switches its working regions. As the load pressure difference between two hydraulic chambers decrease below a point the shuttle valve starts to open and actuator dynamics gets much more complex, where in this study these complexities are not focused, including flows from accumulator to the chambers depending on the shuttle valve opening. Apart from these points, load pressure estimation includes slight deviations in the transient regions and gives better results for the steady state regions.

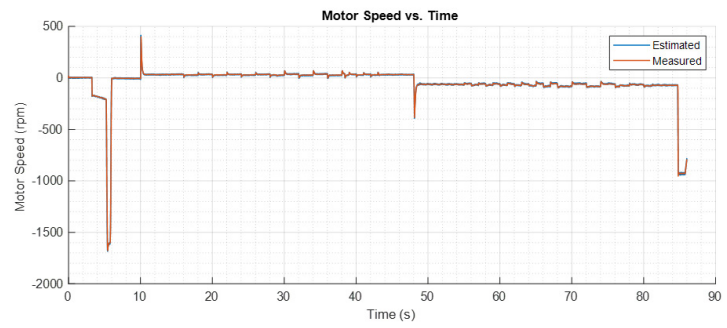

Figure 9. Measured Motor Speed and the Motor Speed Estimation for the EHA.

The state observer gives accurate results for the estimation of the servo motor speed as can be seen in Fig. 9. This is also an expected result since feedback is available for this state variable. In addition to the state estimations, the comparison for the disturbance force estimation is also given with the following figures.

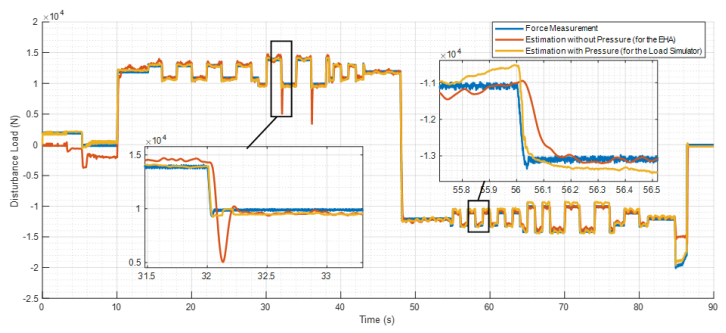

Figure 10. Disturbance Force Estimation (with \& without Pressure Feedback) and the Measured Disturbance.

Considering the results for the disturbance estimation, it could be concluded that the designed disturbance observer gives good estimation results for the steady state cases as can be seen in Fig. 10. Though, there are some deviations from the measured force between the disturbance estimation without using pressure feedback especially in transient regions (for example around 0.2-0.3 second difference in rise time), the estimation still reflects the disturbance dynamics with an adequate level of accuracy.

After about 7-8 bar load pressure, the disturbance and the state observer start to give much better results. This is an expected situation since the shuttle valve inside the EHA system fully opens after about 7-8 bar differential pressure and in the observer dynamics the shuttle valve is assumed to be fully opened to one side.

\section{Test Scenario and Results for Jamming}

Several faulty jamming cases are simulated in the experimental setup and the detection performance of the proposed method is analyzed in this subsection. The most critical and difficult cases in terms of detection and identification of jamming are at low deflection signals where the reference position input to the actuator is quite low especially in cruise (steady state flight) condition. Therefore, low amplitude input signals around the jammed position are inserted to the control system. To simulate several different cases, both sinusoidal and sawtooth signals are used in jamming conditions. In order not to cause an excessive sudden increase in the load and in order not to damage the setup, step signals are not preferred for jamming simulations. It should be noted here that this study basically focuses on the detection of jamming cases for a test setup on ground before any implementation on a real flight test with more realistic pilot (or flight control computer) input behavior. Therefore, generic input signals, whose amplitude and frequency can be varied easily, like sawtooth and sinusoidal waves are used. In this way, amplitude and frequency ranges of actuator input signals under which the jamming cases are observed can be realized. The test scenario is applied under both jammed and not-jammed cases to compare the performance of the developed FDD system under faulty and non-faulty cases. The test cases investigated are listed in Table 3. Amplitude and frequency values of the selected reference position signals are presented in the 5th column of the table. Since the actuator input demand is relatively low in cruise condition with the control surface being quite close to its neutral position, amplitudes of $0.25 \mathrm{~mm}, 0.5 \mathrm{~mm}$ and $1 \mathrm{~mm}$ are chosen. To reflect different demand behaviors of the actuator position, sinusoidal and sawtooth signals with two different frequency values are used. All of the faulty jamming conditions are also simulated for the not-jammed nominal case. For all of the jamming conditions in the following table, a disturbance load of $14000 \mathrm{~N}$ is applied as this value is very close to the maximum force 
Table 3. Test Cases for Jamming.

\begin{tabular}{|c|c|c|c|c|c|}
\hline Test Case & Condition & Initial Condition & Input Signal & Signal Amplitude and Frequency & $\begin{array}{c}\text { Disturbance } \\
\text { Load }\end{array}$ \\
\hline 1 & Jammed & $50 \mathrm{~mm}$ & Sine Wave & $0.25 \mathrm{~mm}, 2 \mathrm{~Hz}$ & $14000 N$ \\
\hline 2 & Notn-Jammed & $50 \mathrm{~mm}$ & Sine Wave & $0.25 \mathrm{~mm}, 2 \mathrm{~Hz}$ & $4000 N$ \\
\hline 3 & Jammed & $50 \mathrm{~mm}$ & Sine Wave & $0.5 \mathrm{~mm}, 1 \mathrm{~Hz}$ & $14000 N$ \\
\hline 4 & Notn-Jammed & $50 \mathrm{~mm}$ & Sine Wave & $0.5 \mathrm{~mm}, 1 \mathrm{~Hz}$ & $4000 N$ \\
\hline 5 & Jammed & $50 \mathrm{~mm}$ & Sine Wave & $1 \mathrm{~mm}, 0.5 \mathrm{~Hz}$ & $14000 N$ \\
\hline 6 & Notn-Jammed & $50 \mathrm{~mm}$ & Sine Wave & $1 \mathrm{~mm}, 0.5 \mathrm{~Hz}$ & $4000 N$ \\
\hline 7 & Jammed & $50 \mathrm{~mm}$ & Sawtooth & $0.25 \mathrm{~mm}, 2 \mathrm{~Hz}$ & $14000 N$ \\
\hline 8 & Notn-Jammed & $50 \mathrm{~mm}$ & Sawtooth & $0.25 \mathrm{~mm}, 2 \mathrm{~Hz}$ & $4000 N$ \\
\hline 9 & Jammed & $50 \mathrm{~mm}$ & Sawtooth & $0.5 \mathrm{~mm}, 0.5 \mathrm{~Hz}$ & $14000 N$ \\
\hline 10 & Notn-Jammed & $50 \mathrm{~mm}$ & Sawtooth & $0.5 \mathrm{~mm}, 0.5 \mathrm{~Hz}$ & $4000 N$ \\
\hline 11 & Jammed & $50 \mathrm{~mm}$ & Sawtooth & $1 \mathrm{~mm}, 0.5 \mathrm{~Hz}$ & $14000 N$ \\
\hline 12 & Notn-Jammed & $50 \mathrm{~mm}$ & Sawtooth & $1 \mathrm{~mm}, 0.5 \mathrm{~Hz}$ & $4000 N$ \\
\hline
\end{tabular}

that can be measured by the load cell in the test setup. Not-jammed cases are simulated under a load of $4000 \mathrm{~N}$.

Experiments are performed based on the test scenario in Table 3. Jamming cases are tried to be simulated under the counter load by the load simulator using a mechanical locking assembly. By this way, EHA piston is kept at the desired position. Reference and measured position, rate of the estimated disturbance, recursive variance calculation of disturbance rate, moving average of the tracking error and the eventual output of the developed FDD method, which is the fault signal, are plotted in the results of the test cases. Moving average of the position tracking error, disturbance rate and its variance are given including both jammed and not-jammed cases in the same figures. Note that both jamming and unjamming conditions are simulated by cancelling integral gain of the controller. Therefore, developed FDD method is analyzed under proportional controller for all jammed and not-jammed conditions. Disturbance loads of $14 \mathrm{kN}$ and $4 \mathrm{kN}$ are applied (at $\mathrm{t}=19 \mathrm{~s}$ ) for the jammed and not-jammed cases, respectively. After the counter load (dis-

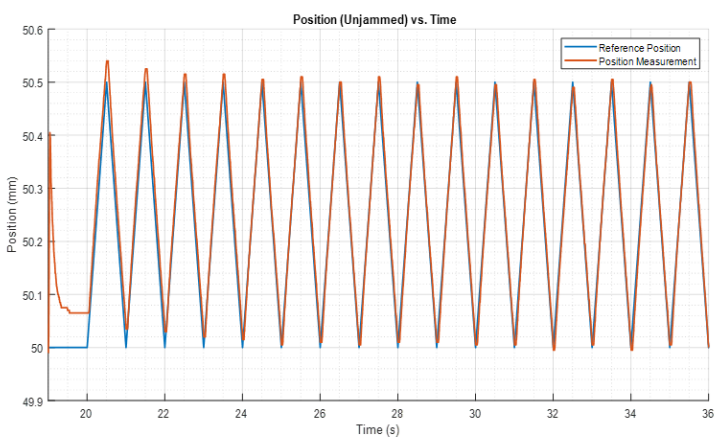

Figure 11. EHA Position Response under the Not-jammed Case for test cases 9. turbance), the reference position is inserted to the EHA at $\mathrm{t}=20 \mathrm{~s}$. Results are given for just one set of test case (Test Cases 9\&10) in the following figures.

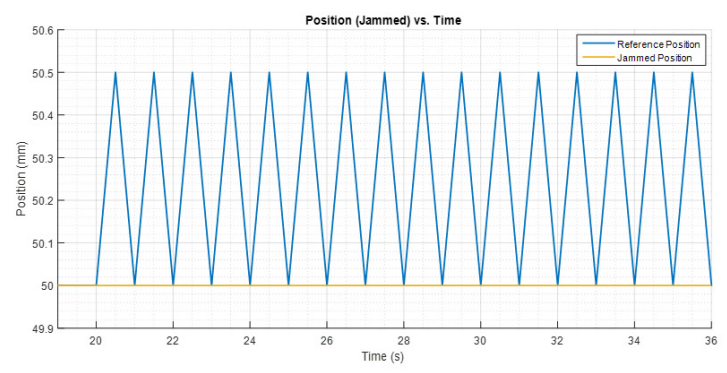

Figure 12. EHA Position Response under the Jammed Cases for test cases10.

Several remark regarding the given plots for the fault detection and diagnosis of jamming failures are highlighted as follows:

Position responses of the EHA under jammed and notjammed cases are as expected. Because of the high disturbance load $(14 \mathrm{kN})$ the actuator cannot track the given position input as can be seen in Fig. 12. Whereas small tracking error occurs (Fig. 11) for the not-jammed case due to the considerably lower disturbance load $(4 \mathrm{kN})$. Note that control system is the same for all jammed and not-jammed cases, so the only difference is created by changing the external disturbance load by means of counter loading and the mechanical locking which have been mentioned before. Rate of the estimated disturbance load seems quite noisy and it is difficult to extract valuable information about the faults from Fig. 13. Therefore, the variance of this disturbance rate is calculated in Fig. 14. There is a quite bit of difference between the jammed cases and the not-jammed ones. High 
position tracking errors are inevitable for jamming cases because of the low disturbance rejection characteristics under mechanical locking or counter loading. The deviation between the tracking error for the not-jammed case and the jammed cases is quite large as expected. The fault is identified (Fig. 16) in a considerably small-time interval for this test case because of the high deviations in the indicators (disturbance rate and the tracking error) between jammed and not-jammed conditions. For all of the simulated test cases, developed FDD system gives very similar results for the fault indicators, detection time and deviations between jamming and unjamming conditions. Using the developed FDD method, all of the failure cases could be identified under 1s. It should be noted that detection time of the faults strictly depends on the chosen threshold values in the fault identification step. The higher the threshold value is, the longer it takes the system to identify the jamming cases. In order to be more robust against false alarms, higher threshold might be chosen but this would considerably increase the lag between the occurrence of the error and the detection time. Overall results are summarized in the following table.

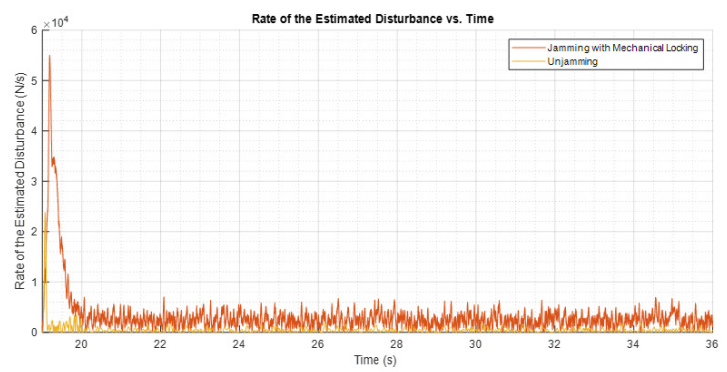

Figure 13. Rate of the Estimated Disturbance for test cases 9 and 10.

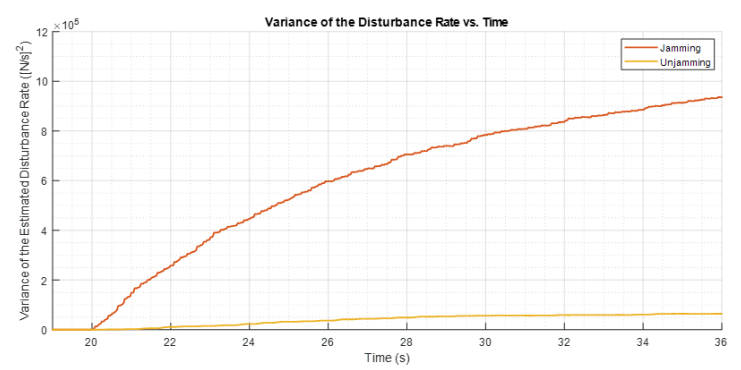

Figure 14. Variance of the Estimated Disturbance Rate for test cases 9 and 10.

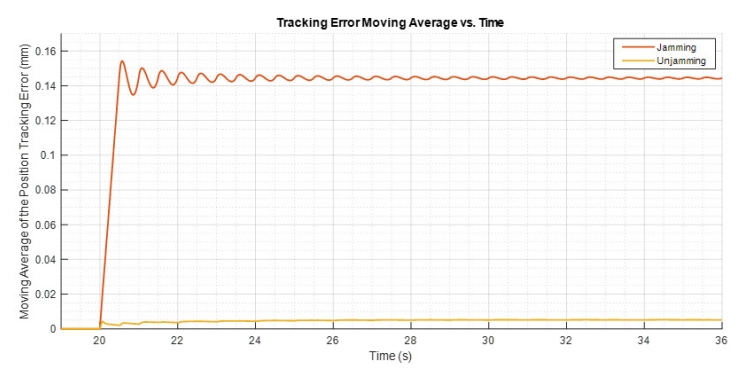

Figure 15. Moving Average of the Position Tracking Error for test cases 9 and 10.

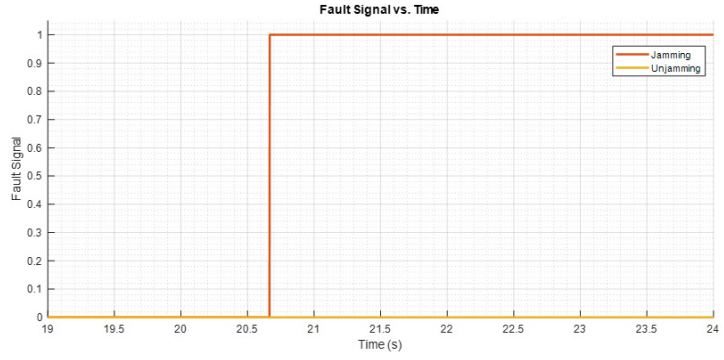

Figure 16. Generated Fault Signal for test cases 9 and 10.

Considering the results provided in Table 4 and 5 , a number of comments can be made regarding failure dynamics, fault detection performance and the overall effectiveness of the developed methodology. A single threshold value is selected in the developed FDD algorithm for both the moving average of the tracking error and the variance of the estimated disturbance rate. Yet, a single threshold set could give comparably good detection times. Maximum fault detection time, $0.68 \mathrm{~s}$, occurs at the amplitude of $0.25 \mathrm{~mm}$. We assumed that an actuator stroke of $0.25 \mathrm{~mm}$ nearly corresponded to a minimum surface deflection of a typical flight control surface. Since the detection time would decrease as the given input is increased at the time of jamming, this $1.26 \mathrm{~s}$ of detection time might be considered as the maximum detection time for a jamming failure of a typical flight control surface.

\section{DISCUSSION AND CONCLUSION}

In this paper, a threshold-based fault detection and diagnosis (FDD) method is developed for jamming. The method is tested on an experimental setup. In order to estimate the state variables correctly, firstly system dynamics of the EHA is modelled. Then, the state and disturbance observers are designed to estimate both unknown system states and the disturbance load.

The whole FDD method is based on two indicators. One indicator is selected as the variance of the time rate of change of the disturbance. When this variance exceeds a predefined threshold value then the second indicator regarding the fault is checked. This indicator is the moving average of the tracking error. In order to test the performance of the proposed FDD method, a fault scenario is created. Low amplitude sine and sawtooth waves with different frequency values around a specific actuator position are taken as the reference inputs. After considering the results, it is realized that the disturbance rejection characteristics of the actuator has a great influence on the fault detection performance of the designed method. Depending on the disturbance rejection of the actuator, its estimated disturbance rate under the jamming case could diverge rapidly from the not-jammed nominal condition. 
Table 4. Summary of the Results for the Sine Wave Inputs.

\begin{tabular}{|c|c|c|c|c|}
\hline Test Case & Input Signal & $\begin{array}{c}\text { Variance of the } \\
\text { Estimated Disturbance } \\
\left([N / s]^{2}\right)\end{array}$ & $\begin{array}{l}\text { Moving Average of the } \\
\text { Tracking Error }(\mathrm{mm})\end{array}$ & $\begin{array}{l}\text { Detection } \\
\text { Time (s) }\end{array}$ \\
\hline $1-2$ & $0.25 \mathrm{~mm}$ Sine & $\begin{array}{c}7.2 \times 10^{5}-\text { Jammed } \\
6 \times 10^{4}-\text { Not-jammed }\end{array}$ & $\begin{array}{c}0.09 \text {-Jammed } \\
0.025 \text {-Not-jammed }\end{array}$ & 0.62 \\
\hline $3-4$ & $0.50 \mathrm{~mm}$ Sine & $\begin{array}{c}9.1 \times 10^{5}-\text { Jammed } \\
5 \times 10^{4}-\text { Not jammed }\end{array}$ & $\begin{array}{c}0.17 \text {-Jammed } \\
0.022-\text { Not jammed }\end{array}$ & 0.51 \\
\hline $5-6$ & $1.00 \mathrm{~mm}$ Sine & $\begin{array}{c}10 \times 10^{5}-\text { Jammed } \\
5 \times 10^{4}-\text { Not jammed }\end{array}$ & $\begin{array}{c}0.28 \text {-Jammed } \\
0.02-\text { Not jammed }\end{array}$ & 0.47 \\
\hline
\end{tabular}

Table 5. Summary of the Results for the Sawtooth Wave Inputs.

\begin{tabular}{|c|c|c|c|c|}
\hline Test Case & Input Signal & $\begin{array}{c}\text { Variance of the } \\
\text { Estimated Disturbance } \\
\left([\mathrm{N} / \mathrm{s}]^{2}\right)\end{array}$ & $\begin{array}{l}\text { Moving Average of the } \\
\text { Tracking Error }(\mathrm{mm})\end{array}$ & $\begin{array}{c}\text { Detection } \\
\text { Time (s) }\end{array}$ \\
\hline $7-8$ & $\begin{array}{l}0.25 \mathrm{~mm} \\
\text { Sawtooth }\end{array}$ & $\begin{array}{c}6.6 \times 10^{5}-\text { Jammed } \\
6.5 \times 10^{4}-\text { Not jammed }\end{array}$ & $\begin{array}{c}0.06 \text {-Jammed } \\
0.023 \text {-Not-jammed }\end{array}$ & 0.68 \\
\hline $9-10$ & $\begin{array}{l}0.50 \mathrm{~mm} \\
\text { Sawtooth }\end{array}$ & $\begin{array}{c}9 \times 10^{5}-\text { Jammed } \\
6 \times 10^{4}-\text { Not jammed }\end{array}$ & $\begin{array}{c}0.14 \text {-Jammed } \\
0.02-\text { Not jammed }\end{array}$ & 0.66 \\
\hline $11-12$ & $\begin{array}{l}1.00 \mathrm{~mm} \\
\text { Sawtooth }\end{array}$ & $\begin{array}{l}9.6 \times 10^{5}-\text { Jammed } \\
6 \times 10^{4}-\text { Not jammed }\end{array}$ & $\begin{array}{c}0.23 \text {-Jammed } \\
0.025-\text { Not jammed }\end{array}$ & 0.62 \\
\hline
\end{tabular}

At the end, the results are given for one of the test cases with jamming and without jamming Fig.s 11-16. Faults are successfully detected (Tables 4-5) for all of the jammed cases and no false positives for not-jammed cases. The detection times are quite low, less than one second for each case which is considerably lower than the values achieved by different methods like LPV model-based detection method where the mean detection and identification time of jamming is $3.57 \mathrm{~s}$ and maximum detection time of $10.65 \mathrm{~s}$ [6]. Last but not the least, several aspects of this study should be addressed before considering any real time flight control system implementation. First point is that every control surface actuator has its own dynamics with its parameters. Therefore, a high-fidelity actuator model needs to be constructed according to the specific architecture selected for the concerning flight control application. Then, realistic actuator inputs shall be generated through either real pilot inputs or processed inputs via a dedicated flight control computer. Taking these points into consideration, the developed method in this paper might be implemented to a flight control application with minimal modifications.

\section{References}

1. Zolghadri, A., Cieslak, J., Efimov, D., Henry, D., Goupil, P., Dayre, R., Leberre, H.. Signal and model-based fault detection for aircraft systems. IFAC-PapersOnLine (2015), 48(21), 1096-1101.

2. Ossmann, D., \& Varga, A. Detection and identification of loss of efficiency faults of flight actuators. Paper presented at the Conference on Control and Fault-Tolerant Systems (SysTol), October. 2013.

3. Cieslak, J., Efimov, D., Zolghadri, A., Gheorghe, A., Goupil, P., \&
Dayre, R. A Method for Actuator Lock-in-place Failure Detection in Aircraft Control Surface Servo-loops. IFAC Proceedings Volumes, 47(3), 10549-10554, 2014.

4. Model-Based Approaches for Fast and Robust Fault Detection in an Aircraft Control Surface Servo Loop: From Theory to Flight Tests [Applications of Control]. IEEE Control Systems,33(3), 20-84, 2013.

5. Jayakumar, M., \& Das, B. Diagnosis of Incipient Sensor Faults in a Flight Control Actuation System. 2006 SICE-ICASE International Joint Conference, 2006.

6. Varga, A., Ossmann, D., Goupil, P., \& Sabot, G. Verification and validation of a FDD system for identification of aircraft control surface jamming*. IFAC Proceedings Volumes, 46(19), 84-89, 2013.

7. Narendra, K., Balakrishnan, J., Adaptive control using multiple models. IEEE Trans. Automat. Control 42, 171-187, 1997.

8. Çalışkan, H., Balkan, T., and Platin, B. E. "A Complete Analysis and a Novel Solution for Instability in Pump Controlled Asymmetric Actuators." ASME. J. Dyn. Sys., Meas., Control. September, 137(9): 091008, 2015.

9. H. Çalışkan. "Development and Control of a Single Rod Electro Hydrostatic Actuator" Ph.D. Thesis, Middle East Technical University, Mechanical Engineering Department, 2015.

10. Won, D., \& Kim, W. Disturbance Observer based backstepping for position control of electro-hydraulic systems. International Journal of Control, Automation and Systems, 13(2), 488-493, 2015.

11. W. Chen, D. Balance, P. Gawthrop, and J.O'Reilly. 'A nonlinear disturbance observer for robotic manipulators," IEEE Trans. Ind. Electron., vol. 47, no. 4, pp. 932-938, 2000.

12. Maré Jean-Charles. Aerospace Actuators 1 Needs, Reliability and Hydraulic Power Solutions. UK, 2016.

13. Welford, B.P. Note on a method for calculating corrected sums of squares and products. Technometrics, 4, 419-420, 1962.

14. H. U. Akova. "Design, Construction and Control of an ElectroHydraulic Load Simulator for Testing Hydraulic Drives," M.Sc. Thesis, Middle East Technical University, Mechanical Engineering Department, 2014. 